\title{
Review of: "A novel method for extraction, quantification, and identification of microplastics in CreamType of cosmetic products"
}

Weiqing Zhou

Potential competing interests: The author(s) declared that no potential competing interests exist.

This study provided a chemical method with a high digestion efficiency for microplastics in the cleansing creams. As we know, the detection and analysis methods of microplastics with the particle size above $20 \mu \mathrm{m}$ have been widely investigated. For example, $\mathrm{KOH}$ digestion method has been reviewed in the paper “MUNNO K, HELM P A, JACKSON D A, et al. Impacts of temperature and selected chemical digestion methods on microplastic particles. Environmental Toxicology and Chemistry, 2018, 37(1): 91-98."

PE microspheres with the particle size of 90-106 $\mu \mathrm{m}$ was studied in this work. But I want to know the digestion efficiency of the optimized chemical method for smaller microplastics (smaller than $20 \mu \mathrm{m}$ ) and especially even nano-plastics, which can be added in this research. 